\title{
Die kommunale Spielapparatesteuer und der bundesstaatliche Finanzausgleich
}

\author{
Das Bundesverfassungsgericht hat in der Vergangenheit Urteile zum föderalen Finanzausgleich \\ in Deutschland gefällt. Eine wichtige Frage war, welche Steuern Teil der Umverteilung im \\ föderalen Finanzausgleich sein sollten. Das Gericht hat Kriterien entwickelt, anhand derer \\ eine Entscheidung möglich ist. Auf Grundlage dieser Kriterien lassen sich Steuern, die Teil \\ des föderalen Finanzausgleichs sind, mit der kommunalen Spielapparatesteuer vergleichen, \\ die dort noch nicht berücksichtigt ist. Der Vergleich zeigt, dass die Spielapparatesteuer in \\ den föderalen Finanzausgleich einbezogen werden sollte, da ihre Einnahmen unter anderem \\ wesentlich höher sind als diejenigen von bereits berücksichtigten Steuern.
}

Im Rahmen des bundesstaatlichen Finanzausgleichs sollen die Einnahmen der Länder möglichst umfassend berücksichtigt werden, damit die bereitgestellten finanziellen Mittel tatsächlich die bestehenden Finanzkraftunterschiede mindern helfen. Das Bundesverfassungsgericht musste in der Vergangenheit mehrmals darüber entscheiden, welche Einnahmen einzubeziehen sind und hat zu diesem Zweck einen Kanon an Beurteilungskriterien entwickelt. Erstaunlich ist in diesem Zusammenhang, dass die Spielapparatesteuer (Synonym: Spielautomatensteuer) als eine Form der kommunalen Vergnügungsteuer in den neuesten Urteilen des Gerichts nicht berücksichtigt wurde. Das Aufkommen der Spielautomatensteuer ist deutlich höher als jenes der Bier- oder Feuerschutzsteuer. Es fällt auch ungleichmäßiger in den Bundesländern an. Damit sind zwei von den Kriterien erfüllt, die das Bundesverfassungsgericht zur Beurteilung von Einnahmen verwendet hat.

\section{Verfassungsrechtliche Prüfungen}

Bei den verfassungsgerichtlichen Auseinandersetzungen im Zusammenhang mit Regelungen des bundes-

(C) Der/die Autor(en) 2020. Open Access: Dieser Artikel wird unter der Creative Commons Namensnennung 4.0 International Lizenz (https:// creativecommons.org/licenses/by/4.0/deed.de) veröffentlicht.

Open Access wird durch die ZBW - Leibniz-Informationszentrum Wirtschaft gefördert.

Prof. Dr. habil. Michael Broer lehrt Volkswirtschaftslehre an der Hochschule Ostfalia in Wolfsburg. staatlichen Finanzausgleichs ging es zuletzt vor allem um die Ausgleichsquoten und darum, in welchem Umfang die bisher berücksichtigten kommunalen Steuern einbezogen werden sollen (Bayerische Staatsregierung, 2013; Kube und Seiler, 2013; Feld und Schnellenbach, 2013). ${ }^{1}$ Keine Rolle spielte hingegen in den jüngsten Urteilen der Kreis der einzubeziehenden Einnahmen, obwohl sich in der Vergangenheit das Bundesverfassungsgericht mit dieser Frage mehrfach befassen musste. So ging es in den ersten beiden Klagen zum Länderfinanzausgleich (LFA) unter anderem auch um die einzubeziehenden Einnahmen und den prozentualen Umfang ihrer Berücksichtigung. Als Folge der Gerichtsurteile galten die Feuerschutz-, die Grunderwerbsteuer und die Spielbankabgabe grundsätzlich und die Förderabgabe ${ }^{2}$ in voller Höhe als ausgleichsrelevant und damit als im LFA zu berücksichtigen (Bundesverfassungsgericht, 1992, 1986).

Im Zusammenhang mit diesen beiden grundsätzlichen Entscheidungen hat das Bundesverfassungsgericht Kriterien entwickelt, auf deren Grundlage ermittelt werden kann, ob Einnahmen in den LFA einzubeziehen sind. So dürfen Einnahmen für den Fall unberücksichtigt bleiben, dass

1 Das letztgenannte Gutachten ist gleichzeitig Anlage 1 der im März 2013 im Auftrag der Länder Bayern und Hessen durch Kube und Seiler eingereichten Klageschrift (Feld und Schnellenbach, 2013).

2 Die Förderabgabe ist eine Abgabe, die Unternehmen an das jeweilige Bundesland für das Recht entrichten müssen, Bodenschätze auszubeuten; siehe hierzu § 31 Bundesberggesetz (BGBI., 1980), das zuletzt durch Artikel 2 Absatz 4 des Gesetzes vom 20. Juli 2017 (BGBI. I S. 2808) geändert worden ist. 
- „ihr Volumen nicht ausgleichserheblich ist,

- wenn sie in allen Ländern verhältnismäßig pro Kopf gleich anfallen,

- wenn sie als Entgelte oder entgeltähnliche Abgaben lediglich Leistungen des Landes ausgleichen oder

- wenn der Aufwand für die Ermittlung der auszugleichenden Einnahmen zur möglichen Ausgleichswirkung außer Verhältnis steht“ (Bundesverfassungsgericht, 1999).

Der Sinn dieser Kriterien erschließt sich aus den Effekten, die mit der Einbeziehung weiterer Einnahmen verbunden sind. Ist das Aufkommen einer Einnahmenkategorie gering, so ergeben sich geringe Verteilungseffekte. Dies umso mehr, wenn diese Einnahmen auch noch gleichmäBig in den Bundesländern anfallen, da in diesem Fall die Finanzkraft gleichmäßig erhöht wird. In diesem Zusammenhang ist auch noch der Aufwand bei der Erfassung der Einnahmen zu beachten. Sind die Verteilungseffekte gering, der Erfassungsaufwand aber hoch, so sollte folglich auf die Einbeziehung der Einnahmen verzichtet werden.

Im Jahr 1992 verneinte das Bundesverfassungsgericht, dass die kommunale Vergnügungsteuer in den LFA einbezogen werden sollte. Begründet wurde dies damals damit, dass das Aufkommen der Vergnügungsteuer 1989 mit 300 Mio. DM im Vergleich zu den einzubeziehenden Einnahmen aus der Feuerschutzsteuer sowie der Spielbankabgabe zu gering sei (Bundesverfassungsgericht, 1992, Abschnitt E. III. 1. a). 1989 betrug das Aufkommen der Feuerschutzsteuer 372 Mio. DM und jenes der Spielbankabgabe 690 Mio. DM (Bundesrat, 1990, 9). Allerdings hat sich in den vergangenen fast 30 Jahren die Lage deutlich geändert. Für 2018 ergab sich ein Aufkommen aus der kommunalen Vergnügungsteuer von 1071,6 Mio. Euro (Statistisches Bundesamt, 2019, 26). Demgegenüber lag 2018 das Aufkommen aus der Feuerschutzsteuer bzw. der Spielbankabgabe, beide Einnahmen dienten 1992 als Benchmark, bei 467 Mio. Euro bzw. bei 138 Mio. Euro (Bundesministerium der Finanzen [BMF], o. D.).

Die Vergnügungsteuer gehört zu den sogenannten örtlichen Verbrauch- und Aufwandsteuern nach Art. 106 Abs. 6 GG. Bei diesen Steuern dürfen die Gemeinden sowohl die Bemessungsgrundlage als auch den Steuertarif eigenständig bestimmen (BMF, 2011, 138 f.). Werden heute die zuvor genannten Kriterien herangezogen, so wäre nach aktuellem Stand zu prüfen, ob auch die kommunale Vergnügungsteuer nicht doch in den LFA einzubeziehen ist. Bei der Vergnügungsteuer wird zwischen jener auf die Vorführung von Bildstreifen und einer sonstigen Vergnügungsteuer un- terschieden, in die auch die Spielautomatensteuer fällt, die hier betrachtet wird.

\section{Besteuerung von Spielautomaten im Rahmen der Vergnügungsteuer}

Unter der sonstigen Vergnügungsteuer finden sich unterschiedliche Steuern, mit denen Vergnügen unterschiedlicher Art besteuert werden. Ihre Erhebung ist zumeist in den jeweiligen kommunalen Abgabengesetzen der Bundesländer geregelt (z.B. Niedersächsisches Gesetz- und Verordnungsblatt, 2017). Bei aller Unterschiedlichkeit ist eine Konstante festzustellen. In allen Bundesländern, die die Erhebung einer (sonstigen) Vergnügungsteuer vorsehen, erheben die Kommunen eine Steuer, die den Aufwand von Spielautomaten in Form einer Spielautomatenoder Spielapparatesteuer oder Ähnliches belastet. ${ }^{3}$ Trotz des Aufkommens der Vergnügungsteuer von über $1 \mathrm{Mrd}$. Euro im Jahr 2018 gibt es in den amtlichen Statistiken keinerlei Informationen über die Anteile der Erhebungsformen am Aufkommen der sonstigen Vergnügungsteuer. Daher wurden im Rahmen dieser Untersuchung Fragen an Kommunen gestellt, um entsprechende Informationen zu erhalten. Angefragt wurden die Stadtstaaten Hamburg, Bremen (inklusive Bremerhaven) und Berlin sowie jeweils die zwei einwohnerstärksten Städte der übrigen Bundesländer. Aus den vorliegenden Daten ergibt sich ein klares Bild. In allen Städten entstammt der Großteil der Einnahmen der sonstigen Vergnügungsteuer aus der Besteuerung von Spielautomaten. Die Werte liegen zwischen rund $91 \%$ und $100 \%$, im Durchschnitt sind es $97,7 \%{ }^{4}$ $\mathrm{Zu}$ beachten ist noch, dass es sich bei der Besteuerung von Spielapparaten zumeist um Geräte mit Gewinnmöglichkeit handelt. Dabei orientiert sich die Besteuerung an der Brutto- bzw. Nettokasse, denn die Spielautomaten haben mittlerweile ausnahmslos ein manipulationssicheres Zählwerk, nachdem die Bundesregierung und die Automatenwirtschaft 1990 eine entsprechende freiwillige Selbstbeschränkungsvereinbarung getroffen haben. ${ }^{5}$

Da die meisten der hier berücksichtigten Städte mehr als 100.000 Einwohner haben, sind einwohnerschwächere Kommunen in einem geringeren Umfang abgebildet. Die

3 Nur in Bayern dürfen die Kommunen seit 1980 keine Vergnügungsteuer erheben, da innen diese Befugnis mit dem Gesetz zur Abschaffung kommunaler Bagatellsteuern vom 21. Dezember 1979 (GVBI. S. 436) genommen wurde.

4 Bei ausbleibenden Antworten wurden entsprechend der Einwohnerzahl weitere Städte angefragt.

5 Unterrichtung durch die Bundesregierung (1990). Diese Selbstbeschränkung hat dazu geführt, dass Zulassungen für Gewinnspielautomaten ohne manipulationssicheres Zählwerk bis einschließlich 1. Januar 1993 erteilt wurden, weshalb davon auszugehen ist, dass es aktuell allein Geräte mit einem manipulationssicheren Zählwerk gibt. So auch das Bundesverwaltungsgericht (2005). 
hier erfassten Städte (Dessau-Roßlau, Schwerin sowie Neunkirchen) weisen mit Werten zwischen $97,9 \%$ und 99,9\% sogar noch höhere Anteile der Spielapparatesteuer am Vergnügungsteueraufkommen auf als die Großstädte. Dies kann damit erklärt werden, dass die übrigen im Rahmen der Vergnügungsteuer erhobenen Steuern (z. B. solche auf Vorführungen von pornografischen und ähnlichen Filmen oder Bildern - auch in Kabinen -, Striptease-Vorführungen, Peepshows, Sex und Erotikmessen) in kleineren Kommunen eine geringere Rolle spielen dürften als in Großstädten. Insofern wird unterstellt, dass die Einnahmen aus der Besteuerung von Spielautomaten in kleineren, hier kaum betrachteten Kommunen einen ähnlich hohen Anteil an den gesamten Einnahmen aus der Vergnügungsteuer ausmachen. Trotzdem wird für die nachfolgende Analyse noch ein Sicherheitsabschlag auf die empirisch ermittelten Werte vorgenommen, um eine Überschätzung der Einnahmen zu vermeiden. Daher wird nicht der geringste hier ermittelte Wert von rund $91 \%$ für den Anteil der Spielautomatenbesteuerung an den Einnahmen aus der Vergnügungsteuer verwendet. Stattdessen wird unterstellt, dass im Durchschnitt in jedem Flächenland $85 \%$ der tatsächlich erzielten Vergnügungsteuereinnahmen der Besteuerung von Spielapparaten entstammen. Für die Stadtstaaten werden - so vorhanden - die erfragten Werte verwendet, ansonsten ebenfalls $85 \%$ unterstellt (vgl. Abbildung 1).

\section{Ausgleichserhebliches Volumen}

Um beurteilen zu können, ob die Vergnügungsteuer auf Spielautomaten in den LFA einbezogen werden sollte, wird zunächst die Aufkommensentwicklung der Steuer auf Spielautomaten mit den im LFA berücksichtigten Einnahmen verglichen, die das geringste Aufkommen aufweisen. Dies sind für die Landesebene die Feuerschutz- und die Biersteuer sowie die Spielbank- und die Förderabgabe und für die kommunale Ebene die Grundsteuer A. Für den hier analysierten 10-Jahreszeitraum von 2009 bis 2018 zeigt sich, dass das Aufkommen aus der Spielapparatebesteuerung mit über $250 \%$ deutlich gestiegen ist (vgl. Abbildung 1). Mit einem Betrag von $911 \mathrm{Mio}$. Euro übertrifft das Aufkommen aus der Spielapparatesteuer jenes der Biersteuer, welches 655 Mio. Euro im Jahr 2018 betrug, um rund 250 Mio. Euro oder fast $40 \%$. Gegenüber den übrigen hier betrachteten und bereits im LFA berücksichtigten Einnahmen ist die Differenz noch größer. Sollte die vergangene Entwicklung anhalten, dürfte die Relevanz der Spielapparatesteuer noch steigen, auch weil die Einnahmenentwicklung der bereits im LFA erfassten Einnahmen wenig dynamisch ausgefallen ist. Allein die Einnahmen aus der Feuerschutzsteuer (45\%) und der Grundsteuer A (13\%) sind gestiegen, wenn auch deutlich schwächer als das Aufkommen aus der Spielapparatebesteuerung.
Abbildung 1

Aufkommensschwächste Steuern im

Länderfinanzausgleich sowie Spielapparatesteuer

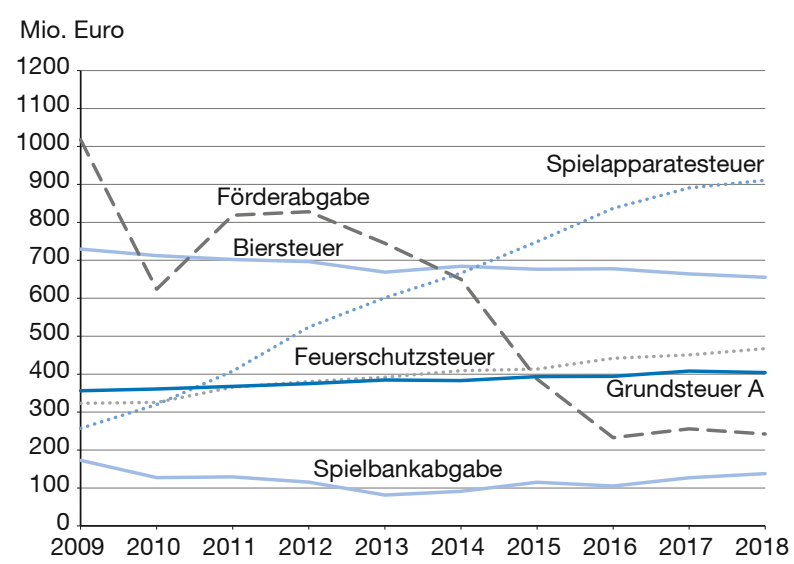

Quelle: eigene Berechnungen und Bundesministerium der Finanzen: Vorläufige Abrechnung des Länderfinanzausgleichs (diverse Jahre); sowie Statistisches Bundesamt (2019), Fachserie 14, Reihe 2, Tab. 2.5, Wiesbaden.

\section{Steueraufkommen je Einwohner}

Neben der Frage der Ausgleichserheblichkeit ist auch die Streuung des Aufkommens je Einwohner für die Entscheidung relevant, ob eine Einnahmenkategorie in den LFA einzubeziehen ist. Daher wird für die hier untersuchten Einnahmen zunächst für jedes Land das Aufkommen je Einwohner ermittelt. Für die Stadtstaaten wird bei dieser Betrachtung das tatsächliche Aufkommen aus der Spielapparatesteuer verwendet, bei den Flächenländern wird wiederum für die Spielapparatesteuer ein Anteil von $85 \%$ am Vergnügungsteueraufkommen unterstellt.

Die sich auf Basis dieser Vorgehensweise ergebenden absoluten Unterschiede zwischen den verschiedenen Einnahmen sind sehr groß. So liegt das maximale Aufkommen je Einwohner bei der Biersteuer bei 24,6 Euro (Bremen) um 21,8 Euro über dem Minimalwert von 2,8 Euro (Hessen). Bei der Feuerschutzsteuer betragen die Unterschiede zwischen den beiden Extremwerten (Hamburg: 6,5 Euro/Kopf und Berlin 4,4 Euro/Kopf) nur 2,1 Euro. Bei der Besteuerung von Spielapparaten erzielt Bremen (inklusive Bremerhaven) je Einwohner mit 28,9 Euro mehr als das zehnfache des Betrags von Brandenburg (2,8 Euro je Einwohner). Aufgrund dieser großen absoluten Differenzen wird jeweils der Maximalwert einer Einnahmenkategorie auf 100 normiert, die übrigen Länderwerte entsprechend dazu ins Verhältnis gesetzt und dann nach absteigender Reihenfolge sortiert (vgl. Abbildung 2). Bei der Feuerschutzsteuer beträgt das Pro-Kopf-Aufkommen mindestens $67,4 \%$ des erzielten 
Maximalwerts. Bei den anderen Einnahmen sind die Abweichungen zwischen diesen beiden Werten deutlich größer. Das Minimum liegt zwischen 11,4\% (Biersteuer) und 0\% (Förderabgabe). Die Spielapparatesteuer erfüllt also das quantitative Kriterium. Ihr Aufkommen verteilt sich deutlich ungleichmäßiger auf die Bundesländer als z.B. jenes aus der Feuerschutzsteuer, die wiederum in den LFA eingeht.

\section{Qualitative Kriterien}

Abschließend sollen die Kriterien drei und vier betrachtet werden: Gleichen die Entgelte oder entgeltähnlichen Abgaben lediglich Leistungen des Landes aus? Oder ist der Aufwand für die Ermittlung der auszugleichenden Einnahmen zu hoch? Da es sich bei der Vergnügungsteuer auf Spielautomaten nicht um Entgelte oder Ähnliches handelt, spricht dieses Kriterium nicht gegen die Einbeziehung dieser Steuer in den LFA. Einzig bei der zweiten Frage ist keine einfache Antwort möglich. Zwar ist das Aufkommen der Vergnügungsteuer auf Spielautomaten deutlich höher als die hier zu Vergleichszwecken herangezogenen Einnahmen, dafür ist die Zahl der Bundesländer mit 16 deutlich geringer als die Zahl der Kommunen in Deutschland. Allerdings werden auch bei der Grundsteuer A für alle Kommunen in Deutschland die Bemessungsgrundlage, die Steuersätze und das Aufkommen für die Normierung erfasst, und dies bei einem Gesamtaufkommen von rund 404 Mio. Euro. Im Vergleich dazu hat die Spielapparatesteuer immerhin ein Aufkommen von rund 911 Mio. Euro. Zudem ist zu beachten, dass sich die Besteuerung der Spielautomaten an der Brutto- bzw. Nettokasse orientiert, da die Spielautomaten fast ausnahmslos ein manipulationssicheres Zählwerk haben. Auf Basis der Einnahmen und der Steuersätze könnte somit relativ einfach eine Normierung vorgenommen werden. Insofern dürfte wohl auch das Kriterium der Erfassungskosten nicht dagegen sprechen, die Vergnügungsteuer auf Spielautomaten in den bundestaatlichen Finanzausgleich einzubeziehen.

Das Problem der hohen Erfassungskosten zum Zwecke der Normierung des Aufkommens aus der Spielapparatesteuer für den LFA könnte allerdings auch vermieden werden, wenn diese wie die bereits im LFA erfasste Spielbankabgabe behandelt werden würde. Letztere geht mit dem tatsächlichen Aufkommen in den LFA ein. Eine Normierung der Einnahmen der Spielbankabgabe unterbleibt, obwohl z. B. die maximalen Abgabensätze in den Ländern zwischen $20 \%$ (Bremen) und $80 \%$ (Mecklenburg-Vorpommern) liegen (Broer, 2018). Bei solch großen Unterschieden gilt eigentlich eine Normierung als notwendig und aufgrund der geringen Zahl an Bundesländern auch als möglich. Da seit Jahrzehnten auf die Normierung der Spielbankabgabe verzichtet wird, könnte sich diese Vorgehensweise auch bei der Vergnügungsteuer auf Spielautomaten anbieten.

\section{Abbildung 2}

Einnahmen je Einwohner bei den aufkommensschwächsten Einnahmen, 2018

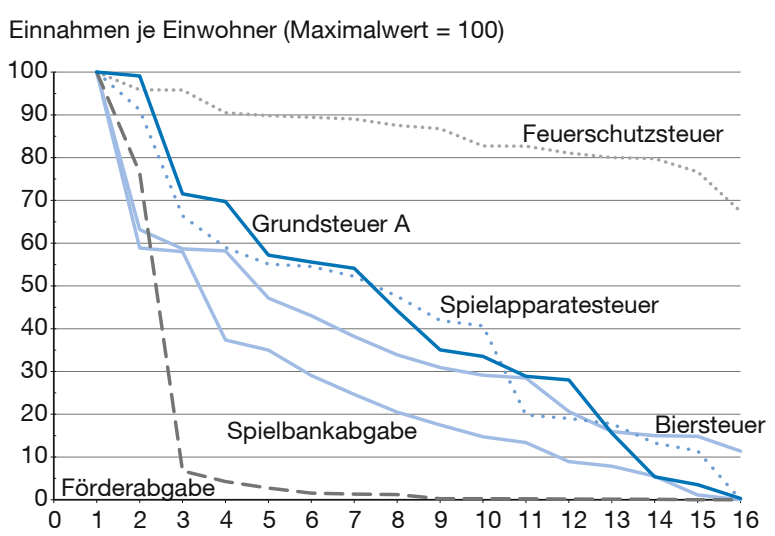

${ }^{1} \mathrm{Da}$ in Bayern keine Vergnügungsteuer erhoben werden darf, fehlt bei dieser Steuer der 16. Wert.

Quelle: eigene Berechnungen und BMF (2019).

\section{Umverteilungswirkung}

Welche finanziellen Effekte hätte eine Berücksichtigung der kommunalen Spielapparatesteuer im bundestaatlichen Finanzausgleich? Hier müssten die Einnahmen normiert werden, was schwierig ist, da derzeit amtliche Statistiken über die Steuersätze in den Kommunen nicht vorliegen. In Analogie zur Spielbankabgabe könnte auf eine Normierung der Einnahmen aus der kommunalen Spielapparatesteuer verzichtet und das tatsächliche Aufkommen verwendet werden. Dies wird nachfolgend geschehen, wobei für alle Bundesländer unterstellt wird, dass $85 \%$ des Aufkommens der Vergnügungsteuer auf die Spielapparatesteuer entfallen (vgl. Abbildung 1). ${ }^{6}$ In diesem Fall können die Verteilungseffekte der Einbeziehung der Spielapparatesteuer mit einem Volumen von 911 Mio. Euro sowohl beim LFA als auch bei den Bundesergänzungszuweisungen (BEZ) - sowohl den allgemeinen BEZ als auch den BEZ zum Ausgleich kommunaler Finanzschwäche - ermittelt werden. Da die Einwohnerzahlen der Bundesländer stark differieren - 17,9 Mio. in NordrheinWestfalen gegenüber 0,7 Mio. in Bremen, werden nachfolgend die finanziellen Effekte je Einwohner betrachtet.

Durch die Einbeziehung der nicht normierten Einnahmen der kommunalen Spielapparatesteuer können bei einzelnen Ländern über alle drei der zuvor genannten Vertei-

6 Für die Stadtstaaten liegen die Angaben zu den tatsächlichen Einnahmen aus der Spielapparatesteuer vor, allerdings würde es bei deren Verwendung Verzerrungen gegenüber den Flächenländern geben, bei denen der Anteil der Spielapparatesteuer pauschal mit $85 \%$ festgelegt wird. Daher wird auch bei den Stadtstaaten so verfahren. 
lungsstufen Mehreinnahmen zwischen 0,2 Euro (Schleswig-Holstein) und 8,9 Euro je Einwohner (Brandenburg) erzielt werden. Die Mindereinnahmen liegen zwischen 1,0 Euro (Hessen) und bis zu 7,1 Euro je Einwohner (Bremen) (vgl. Abbildung 3). Da die Einnahmen aus der Spielapparatesteuer vor allem in den neuen Ländern sehr gering ausfallen, verbessert sich bei all diesen Bundesländern die finanzielle Situation, wobei hier der größere Teil des finanziellen Zugewinns auf die BEZ entfällt. Von den zusätzlichen 8,9 Euro je Einwohner, die das Land Brandenburg erhält, entfallen 3,9 Euro auf den LFA und 5,0 Euro auf die BEZ. Von den westdeutschen Bundesländern profitieren die Zahlerländer Bayern und Hamburg sowie die Empfängerländer Berlin und Schleswig-Holstein finanziell von der Einbeziehung der Spielapparatesteuer. Für die übrigen Zahlerländer sowie die finanzschwachen westdeutschen Bundesländer verschlechtert sich die finanzielle Situation. Neben Bremen erleidet das Saarland hohe finanzielle Verluste. Letzteres vor allem wegen der BEZ zum Ausgleich der kommunalen Finanzschwäche, die sich durch die Einnahmen aus der Spielapparatesteuer deutlich mindern.

Auch für den Bund verschlechtert sich die finanzielle Situation, wenn die kommunale Spielapparatesteuer in den bundesstaatlichen Finanzausgleich einbezogen wird, weil vor allem die ostdeutschen Länder aufgrund geringer Einnahmen aus der Spielapparatesteuer zusätzliche Beträge in Form allgemeiner BEZ und BEZ zum Ausgleich der kommunalen Finanzschwäche erhalten. Bremen, Niedersachsen, Rheinland-Pfalz sowie das Saarland erhalten im geringeren Umfang allgemeine BEZ, beim Saarland mindern sich zudem die Zahlungen in Form der BEZ zum Ausgleich der kommunalen Finanzschwäche. Letztlich steigen die Bundesausgaben insgesamt um 40,5 Mio. Euro, wovon 10,2 Mio. Euro auf allgemeine BEZ und 30,3 Mio. Euro auf die BEZ zum Ausgleich für die kommunale Finanzschwäche entfallen.

\section{Zusammenfassung}

Das Bundesverfassungsgericht hat in seinen Urteilen zum Länderfinanzausgleich Kriterien aufgestellt, anhand derer entschieden werden kann, unter welchen Umständen Einnahmen in den LFA einzubeziehen sind. Werden diese Kriterien auf die kommunale Vergnügungsteuer für Spielautomaten angewendet, so zeigt sich, dass die quantitativen Kriterien (Aufkommenshöhe und Ungleichheit der Verteilung zwischen den Ländern) als erfüllt angesehen werden können. Das Aufkommen aus der Vergnügungsteuer für Spielautomaten ist mit einem hier für das Jahr 2018 ermittelten Wert in Höhe von mehr als 911 Mio. Euro deutlich höher als jenes der Biersteuer (655 Mio. Euro), der Feuerschutzsteuer (467 Mio. Euro) oder der Grund-

\section{Abbildung 3}

Gewinne und Verluste aufgrund der kommunalen Spielapparatesteuer ${ }^{1}$

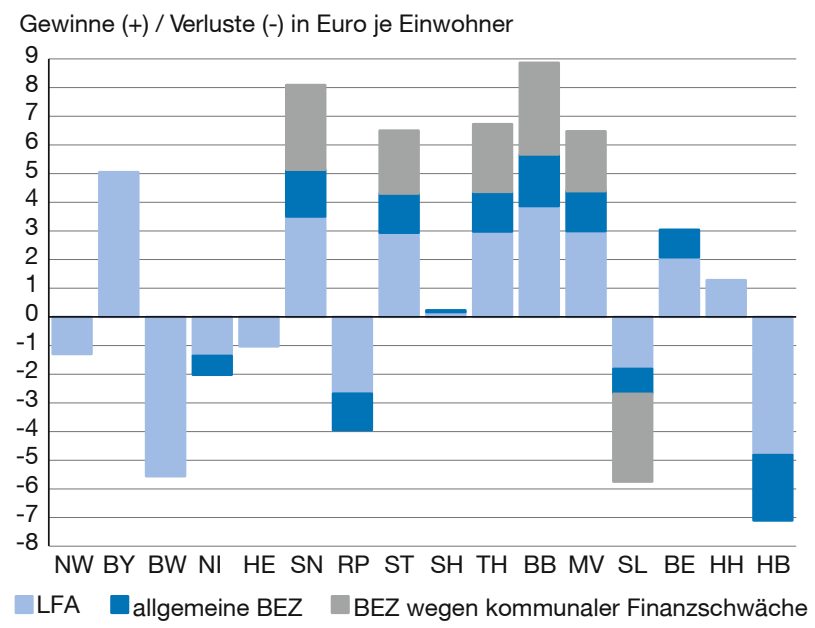

${ }^{1}$ Aufgrund der Berücksichtigung beim Länderfinanzausgleich (LFA) und bei den Bundesergänzungszuweisungen (BEZ).

Anmerkung: NW = Nordrhein-Westfalen, BY = Bayern, BW = BadenWürttemberg, $\mathrm{NI}=$ Niedersachsen, $\mathrm{HE}=$ Hessen, $\mathrm{SN}=$ Sachsen, $\mathrm{RP}=$ Rheinland-Pfalz, ST = Sachsen-Anhalt, SH = Schleswig-Holstein, $\mathrm{TH}=$ Thüringen, $\mathrm{BB}=$ Brandenburg, $\mathrm{MV}=$ Mecklenburg-Vorpommern, $\mathrm{SL}=$ Saarland, BE = Berlin, $\mathrm{HH}=$ Hamburg, $\mathrm{HB}=$ Bremen.

Quelle: eigene Berechnungen sowie Bundesministerium der Finanzen (2019).

steuer A (404 Mio. Euro) sowie der Förder- (242 Mio. Euro) und der Spielbankabgabe (138 Mio. Euro). Letztere werden wie die zuvor genannten Steuern im LFA einbezogen.

Bei der Steuerkraft je Einwohner ist ebenfalls erkennbar, dass die Differenzen bei der Vergnügungsteuer für Spielautomaten größer sind als bei anderen im Finanzausgleich einbezogenen Steuern. So beträgt bei der Feuerschutzsteuer das Aufkommen je Einwohner mindestens $67,4 \%$ des maximalen Pro-Kopf-Aufkommens in einem Bundesland. Bei den anderen Steuern, auch bei der Vergnügungsteuer auf Spielapparate, liegen die Werte für das aufkommenschwächste Bundesland deutlich niedriger. Somit spricht auch die ungleiche Verteilung auf die Länder dafür, die Vergnügungsteuer auf Spielautomaten in den LFA einzubeziehen.

Auch die beiden qualitativen Kriterien des Bundesverfassungsgerichts dürfen bei der Vergnügungsteuer auf Spielautomaten als erfüllt angesehen werden. Die Steuer ist kein Entgelt für andere Leistungen. Auch der Aufwand zur Ermittlung der Einnahmen dürfte allein aufgrund der Einnahmenhöhe nicht unverhältnismäßig sein, zumal 
davon ausgegangen werden kann, dass mittlerweile nur noch Automaten mit einem manipulationssicheren Zählwerk eingesetzt werden. Bei diesen Geräten kann die Steuerbemessungsgrundlage (Brutto- oder Nettokasse) relativ einfach ermittelt werden. Aus diesen Umständen folgt, dass bei der nächsten Reform des Länderfinanzausgleichs geprüft werden sollte, ob die Vergnügungsteuer auf Spielapparate einzubeziehen ist. Dass eine solche Prüfung möglicherweise in gar nicht ferner Zukunft liegt, könnte an der Grundsteuerreform und der dabei im Gesetzentwurf vorgesehenen Öffnungsklausel bei der Steuerbemessungsgrundlage liegen (Gesetzentwurf der Fraktionen der CDU/CSU und SPD, 2019, 131). Vorgesehen ist, bis 2028 einen Mechanismus zu entwickeln, mit dessen Hilfe die Effekte unterschiedlicher Grundsteuerbemessungsgrundlagen, z. B. bodenwert- und flächenorientierte Bemessungsgrundlage (Zimmermann, 2019), auf den Länderfinanzausgleich neutralisiert werden (Finanzausschuss, 2019). Ist absehbar, dass dies nicht gelingen wird, so ist davon auszugehen, dass einzelne Länder in nicht ferner Zukunft vor das Bundesverfassungsgericht ziehen werden. In diesem Zusammenhang sollte dann auch die Berücksichtigung Spielapparatesteuer bei der Finanzkraft der Kommunen im LFA thematisiert werden.

Wird bei der Spielapparatesteuer wie bei der Spielbankabgabe auf eine Normierung des Aufkommens verzichtet, so können die finanziellen Effekte der Einbeziehung der Spielapparatesteuer auf den bundesstaatlichen Finanzausgleich - LFA sowie die BEZ - auf Basis der vorläufigen Abrechnung für 2018 schon derzeit ermittelt werden. Die Gewinne betragen maximal 8,9 Euro je Einwohner (Brandenburg), die Verluste 7,1 Euro je Einwohner (Bremen). Die Ausgaben des Bundes für allgemeine BEZ und BEZ zum Ausgleich der kommunalen Finanzschwäche steigen um rund 40,5 Mio. Euro.

\section{Literatur}

Bayerische Staatsregierung (2013), Ministerpräsident Seehofer und Ministerpräsident Bouffier: „Länderfinanzausgleich muss Anstrengungen
belohnen"/Bayern und Hessen beschließen Klage gegen Länderfinanzausgleich, Pressemitteilung vom 5. Februar.

Broer, M. (2018), Opportunistisches Verhalten der Bundesländer am Beispiel der Spielbankabgabe im Länderfinanzausgleich, in M. Junkernheinrich et al. (Hrsg.), Jahrbuch für öffentliche Finanzen 2019, 427-445.

Bundesgesetzblatt (BGBI, 1980), Teil I, Bundesberggesetz vom 13. August, 1310.

Bundesministerium der Finanzen (BMF) (o.D.), Vorläufige Abrechnung des Länderfinanzausgleichs. Der Finanzausgleich unter den Ländern für die Zeit vom 01.01.2018 - 31.12.2018, https://www.bundesfinanzministerium.de/Content/DE/Standardartikel/Themen/Oeffentliche_Finanzen/ Foederale_Finanzbeziehungen/Laenderfinanzausgleich/vorlaeufigeAbrechnung_2018.pdf?__blob=publicationFile\&v=3 (17. Juni 2020).

Bundesministerium der Finanzen (BMF) (2011), Steuern von A bis Z.

Bundesrat (1990), Verordnung des Bundesministers der Finanzen: Verordnung zur Durchführung des Gesetzes über den Finanzausgleich zwischen Bund und Ländern im Ausgleichsjahr 1989, Drucksache 696/90 vom 8 . Oktober.

Bundesregierung (1990), Bericht der Bundesregierung über Selbstbeschränkungsvereinbarungen der Automatenwirtschaft, Bundestagsdrucksache 11/6224 vom 15. Januar.

Bundesverfassungsgericht (1986), Urteil vom 24.6.1986, http://www.servat. unibe.ch/dfr/bv072330.html (17. Juni 2020).

Bundesverfassungsgericht (1992), Urteil vom 27.5.1992, in Entscheidungen des Bundesverfassungsgerichts, Bd. 86, Tübingen, 148-279.

Bundesverfassungsgericht (1999), Entscheidung 2 BvF 2/98 vom 11.11.1999, Absatz-Nr. 294, http://www.bverfg.de/entscheidungen/ fs19991111_2bvf000298.html 17. Juni 2020).

Bundesverwaltungsgericht (2005), Urteil vom 13.4.2005 - BVerwG $10 \mathrm{C}$ 5.04, Abschnitt II. 2 bb) (2).

Feld, L. P. und J. Schnellenbach (2013), Verzerrungen im bundesstaatlichen Finanzausgleich, Gutachten im Auftrag des Freistaates Bayern und des Landes Hessen, 16.

Finanzausschuss (2019), Bericht des Finanzausschusses (7. Ausschuss) , a) zu dem Gesetzentwurf der Fraktionen der CDU/CSU und SPD- Drucksache 19/11085 - Entwurf eines Gesetzes zur Reform des Grundsteuer- und Bewertungsrechts (Grundsteuer-Reformgesetz - GrStRefG) b) zu dem Gesetzentwurf der Bundesregierungdrucksachen 19/13453, 19/13713 - Entwurf eines Gesetzes zur Reform des Grundsteuer- und Bewertungsrechts (Grundsteuer-Reformgesetz - GrStRefG, Bundestagsdrucksache 19/1415819 vom 10.2019, 5 .

Gesetzentwurf der Fraktionen der CDU/CSU und SPD (2019), Entwurf eines Gesetzes zur Reform des Grundsteuer- und Bewertungsrechts (Grundsteuer-Reformgesetz - GrStRefG), Bundestagsdrucksache, 19/11085 vom 25.6.2019.

Kube, H. und C. Seiler (2013), Antrag im Verfahren der abstrakten Normenkontrolle, 4. März, 47-69.

Niedersächsisches Gesetz- und Verordnungsblatt (2017), § 3 Abs. 2 Niedersächsisches Kommunalabgabengesetz in der Fassung vom 20 . April, Nds. GVBI. Nr. 7/2017, 121.

Statistisches Bundesamt (2019), Fachserie 14, Reihe 2 - 2018, Wiesbaden.

Zimmermann, H. (2019), Grundsteuer: die Qual der Wahl, Wirtschaftsdienst, 99(7), 490-493, https://www.wirtschaftsdienst.eu/inhalt/jahr/2019/ heft/7/beitrag/grundsteuer-die-qual-der-wahl.html (17. Juni 2020).

Title: The Local Entertainment Tax and the Federal Financial Equalisation System in Germany

Abstract: In the past, the federal constitutional court has made judgments about the federal financial equalisation system in Germany, including about taxes should be part of the redistribution of the federal financial equalisation system. The court has developed some criteria e.g. the revenue at all and the per capita revenue. These criteria can be used to compare some taxes that are actually part of the redistribution of the federal financial equalisation system (e.g. beer, gaming casinos and fire protection taxes), with the local slot machine tax (a type of entertainment tax) that is not yet part of the federal financial equalisation system. The comparison shows that the local slot machine tax should be part of the federal system because its revenue is much higher than the revenue of the beer or fire protection tax. JEL Classification: H23, H29, H71, H77 